\title{
Impaired cerebellar functional connectivity in schizophrenia patients and their healthy siblings
}

\section{Guusje Collin ${ }^{1,2}$, Hilleke E. Hulshoff Pol ${ }^{1,2}$, Sander V. Haijma ${ }^{1,2}$, Wiepke Cahn ${ }^{1,2}$, René S. Kahn ${ }^{1,2}$ and Martijn P. van den Heuvel ${ }^{1,2}$}

1 Rudolf Magnus Institute of Neuroscience, Utrecht, Netherlands

${ }^{2}$ University Medical Center Utrecht, Utrecht, Netherlands

\section{Edited by:}

Alex Fornito, University of Melbourne, Australia

\section{Reviewed by:}

John Hart, University of Texas at Dallas, USA

Heather C. Whalley, University of Edinburgh, UK

Alex Fornito, University of Melbourne, Australia

\section{*Correspondence}

Guusje Collin, Department of Psychiatry, University Medical Center Utrecht, A.00.1.12, Heidelberglaan 100, 3584 CX Utrecht, Netherlands. e-mail:g.collin@umcutrecht.nl
The long-standing notion of schizophrenia as a disorder of connectivity is supported by emerging evidence from recent neuroimaging studies, suggesting impairments of both structural and functional connectivity in schizophrenia. However, investigations are generally restricted to supratentorial brain regions, thereby excluding the cerebellum. As increasing evidence suggests that the cerebellum contributes to cognitive and affective processing, aberrant connectivity in schizophrenia may include cerebellar dysconnectivity. Moreover, as schizophrenia is highly heritable, unaffected family members of schizophrenia patients may exhibit similar connectivity profiles. The present study applies resting-state functional magnetic resonance imaging to determine cerebellar functional connectivity profiles, and the familial component of cerebellar connectivity profiles, in 62 schizophrenia patients and 67 siblings of schizophrenia patients. Compared to healthy control subjects, schizophrenia patients showed impaired functional connectivity between the cerebellum and several left-sided cerebral regions, including the hippocampus, thalamus, middle cingulate gyrus, triangular part of the inferior frontal gyrus, supplementary motor area, and lingual gyrus (all $p<0.0025$, whole-brain significant). Importantly, siblings of schizophrenia patients showed several similarities to patients in cerebellar functional connectivity, suggesting that cerebellar dysconnectivity in schizophrenia might be related to familial factors. In conclusion, our findings suggest that dysconnectivity in schizophrenia involves the cerebellum and that this defect may be related to the risk to develop the illness.

Keywords: cerebellum, schizophrenia, siblings, functional connectivity, resting-state fMRI, dysconnectivity

\section{INTRODUCTION}

The cerebellum has long been regarded as a brain structure that is exclusively involved in motor systems, but an increasing body of evidence suggests that is also involved in cognition and emotion (Schmahmann and Caplan, 2006). The cerebellum is thought to influence motor systems by evaluating disparities between intention and action and by adjusting the operation of motor cortices accordingly, through feed-back and -forward loops via the thalamus and pons (Kandel et al., 2000). The cerebellum may be integrated in the neural circuits governing higher cognitive functions in a similar fashion. Cerebellar modulation of cognitive processes was shown in error-related learning and timing, but a more general involvement has also been suggested (Schmahmann, 2000; Andreasen and Pierson, 2008). According to the "cognitive dysmetria" and "dysmetria of thought" models of schizophrenia - a severe psychiatric disorder characterized by hallucinations, delusions, and disintegration of thinking - aberrant cerebellar modulation of information from and to the cerebral cortex may be a part of the pathophysiology of schizophrenia (Andreasen et al., 1998; Schmahmann, 1998).

The notion that schizophrenia involves the aberrant integration of information between anatomically separated brain regions is long-standing (Wernicke, 1906; Bleuler, 1911; Kraepelin, 1919;
Friston, 1998; Stephan et al., 2009a) and is supported by recent neuroimaging studies showing aberrant structural (Assaf and Pasternak, 2008; Bassett et al., 2008; Bassett and Bullmore, 2009; Van den Heuvel et al., 2010; Zalesky et al., 2011) and functional (Lynall et al., 2010; Fornito et al., 2011) connectivity in schizophrenia (Petterson-Yeo et al., 2011; Rubinov and Bassett, 2011). However, the cerebellum is typically excluded from these "whole-brain" analyses of brain connectivity in schizophrenia. Interestingly, some studies using diffusion tensor imaging (DTI) to target specific white matter tracts have shown impaired structural connectivity of the cerebellum (Kanaan et al., 2009; Kyriakopoulos and Frangou, 2009). Furthermore, a few functional connectivity studies in schizophrenia have reported impaired functional integration of the cerebellum (Honey et al., 2005; Kim et al., 2008; Becerril et al., 2011; Repovs et al., 2011). However, it remains unclear whether functional connectivity between the cerebellum and the rest of the brain is affected in schizophrenia, and if so, to what extent. Functional connectivity between anatomically separated brain regions is defined as the temporal dependency of their neural activation patterns and is thought to be reflected, to some extent, by their coherence in spontaneous (resting-state) fluctuations in functional magnetic resonance imaging (fMRI) signal (Friston et al., 1993; Biswal et al., 1995, 1997; Van den Heuvel and Hulshoff Pol, 
2010). Allowing for the investigation of all functional connections of the cerebellum, the present study employs resting-state fMRI to determine the functional connectivity profile of the cerebellum in schizophrenia. Furthermore, as schizophrenia is highly heritable (Sullivan et al., 2003), aberrant connectivity in schizophrenia may also be present in unaffected family members of schizophrenia patients. To determine the familial component of any changes in cerebellar connectivity profiles, these profiles are also investigated in siblings of schizophrenia patients, who share the genetic predisposition for psychosis, but are not (yet) ill. This way, the present study seeks to determine whether impaired cerebellar connectivity might be related to the risk to develop schizophrenia.

\section{MATERIALS AND METHODS PARTICIPANTS}

A total of 62 schizophrenia patients, 67 siblings of schizophrenia patients, and 41 healthy comparison subjects were included in this study. Study participants were recruited at the University Medical Center Utrecht, during a large ongoing cohort in the Netherlands (Genetic Risk and Outcome of Psychosis; GROUP). The study was approved by the affiliated ethical committee. Study participants were between 18 and 60 years of age. All subjects provided written informed consent prior to participation. Subjects with a history of head trauma or major medical or neurological illness were excluded.

Presence or absence of current and lifetime psychopathology was established for all participants, using the Comprehensive Assessment of Symptoms and History (CASH) interview (Andreasen et al., 1992). Schizophrenia patients were eligible for the present study if they met Diagnostic and Statistical Manual of Mental Disorders fourth edition (DSM IV; American Psychiatric Association, 1994) criteria of schizophrenia or related spectrum disorders. Both siblings of schizophrenia patients and healthy comparison subjects could have no history of any psychiatric illness, including substance dependence of abuse; and healthy comparison subjects had no first- or second-degree family members with a lifetime psychotic disorder.

Study participants originated from a total number of 132 families. Within the healthy comparison group, there were two sibling pairs (both healthy control subjects, i.e., without a first- or seconddegree relative with a lifetime psychotic disorder), and within the 67 siblings of patients, there were seven pairs of siblings of a schizophrenia patient and one set of three siblings of a schizophrenia patient. There were no family relationships within the patient group. Between the sibling and patient groups, there were a total number of 27 family-ties [i.e., a schizophrenia patient and (one of) their sibling(s) participated in the study].

For schizophrenia patients, symptom severity was assessed using the positive and negative syndrome scale (PANSS; Kay et al., 1987); Furthermore, the type and daily dose of antipsychotic medication at the time of scanning was recorded, and a haloperidol equivalent dose was calculated using conversion rates (risperidone 0.5:1; olanzapine 1.66:1; quetiapine 25:1; clozapine 33.33:1; aripiprazole 2.5:1; flupenthixol 0.66:1; perphenazine 2.66:1; Kroken et al., 2009). In healthy comparison subjects and siblings of patients, a shortened version of the structured interview of schizotypy-revised (SIS-R; Kendler et al., 1989; Vollema and
Ormel, 2000) was employed [excluding four items: social isolation (last 3 years); antisocial behavior; dysfunction (obligatory activities); and dysfunction (personal caretaking)], to measure the overall severity of schizotypal signs and symptoms on a four-point scale $(0=$ absent, $1=$ mild, $2=$ moderate, $3=$ severe $)$. For all participants, global cognitive functioning, as measured by total IQ, was estimated using four subtests (i.e., Information, Arithmetic, Block design, and Digit symbol coding) of the Dutch version of the Wechsler adult intelligence scale (WAIS; Stinissen et al., 1970).

Finally, statistical testing of group-differences in demographic characteristics was performed using analysis of variance (ANOVA) for continuous and Chi-Square tests for categorical variables. All demographic and clinical characteristics are provided in Table $\mathbf{1 .}$

\section{IMAGE ACQUISITION AND PREPROCESSING}

Resting-state fMRI data were acquired on two $1.5 \mathrm{~T}$ Magnetic Resonance Imaging scanners (Philips Medical Systems, Best, The Netherlands) at the University Medical Center Utrecht, The Netherlands. BOLD time-series were recorded during 9 minutes using a 3D-PRESTO sequence (Van Gelderen et al., 1995; Ramsey et al., 1996; acquisition parameters: TR/TE 21.1/31.1 ms; voxel size $4 \mathrm{~mm} \times 4 \mathrm{~mm} \times 4 \mathrm{~mm})$. Subsequently, a T1 weighted image was acquired for anatomical reference (3D FFE pulse sequence, $\mathrm{TR} / \mathrm{TE}=30 / 4.6 \mathrm{~ms}$, flip-angle $30^{\circ}, \mathrm{FOV} 256 \mathrm{~mm} \times 256 \mathrm{~mm}$, voxel size $1 \mathrm{~mm} \times 1 \mathrm{~mm} \times 1.2 \mathrm{~mm}, 160-180$ contiguous slices; Ramsey et al., 2006). Preprocessing was performed using SPM5 software. In short, all resting-state functional images were registered to the last functional scan to correct for head movements and co-registered with the T1 scan to ensure overlap between the anatomical reference scan and resting-state time-series. The registered functional images were spatially smoothed, using an 8 -mm full width halfmax smoothing kernel. Next, the T1 scan and resting-state timeseries were normalized to standard space, matching the MNI-152 template. To create individual anatomical label maps, the spatially normalized T1 image was overlaid with the automated anatomical labeling (AAL) template (Tzourio-Mazoyer et al., 2002), distinguishing 116 anatomical brain regions. Time-series were corrected for white matter, ventricle, and motion parameters (using regression) and band pass filtered (bandwidth $0.01-0.1 \mathrm{~Hz}$ ) to eliminate low frequency noise and influences of frequencies reflecting possible cardiac or respiratory oscillations.

\section{CEREBELLAR REGION OF INTEREST DEFINITION}

The cerebellum has three anterior-posterior divisions (Kandel et al., 2000). The primary fissure separates the anterior lobe from the posterior lobe and the posterolateral fissure separates the posterior and flocculo-nodular lobe. Three mediolateral regions that are important functionally are distinguished by two longitudinal grooves that define an elevated ridge in the midline known as the vermis and the cerebellar hemispheres on either side of the vermis. Each of the 26 cerebellar regions distinguished by the AAL template was assigned to one of seven cerebellar anatomical regions of interest (ROI; Table 2), including the anterior and posterior vermis, the bilateral anterior and posterior cerebellar hemispheres and the flocculo-nodular lobe (see Figure 1, no. 3; 6; 1 and 2; 4 and $5 ; 7$ respectively). 
Table 1 | Demographic and clinical characteristics.

\begin{tabular}{|c|c|c|c|}
\hline & Healthy control subjects $(N=41)$ & Siblings of patients $(N=67)$ & Schizophrenia patients $(N=62)$ \\
\hline Age, mean (SD) & $30.4(8.8)$ & $29.8(8.0)$ & $31.2(5.8)$ \\
\hline Gender, M/F & $19 / 22$ & $34 / 33$ & $52 / 10^{*}$ \\
\hline Highest degree of education ${ }^{\mathrm{b}}$ (SD) & $5.5(1.4)$ & $5.2(2.1)$ & $4.1(2.1)^{*}$ \\
\hline $1 \mathrm{O}^{\mathrm{c}}(\mathrm{SD})$ & $110.1(15.4)^{*}$ & $102.7(14.9)^{*}$ & $93.7(13.5)^{*}$ \\
\hline Schizophrenia, $N(\%)$ & & & $44(71.0)$ \\
\hline Schizophreniform disorder, $N(\%)$ & & & $3(4.8)$ \\
\hline Schizoaffective disorder, $N(\%)$ & & & $8(12.9)$ \\
\hline Other ${ }^{d}, N(\%)$ & & & 7 (11.3) \\
\hline Schizotypal features, mean (SD) [range] & $0.19(0.18)[0-0.8]$ & $0.17(0.15)[0-0.5]$ & \\
\hline \multicolumn{4}{|l|}{ PANSS symptoms ${ }^{\mathrm{e}}$} \\
\hline \multicolumn{4}{|l|}{ Antipsychotic medication ${ }^{f}$} \\
\hline Atypical, N & & & 43 \\
\hline Risperidone, $N$; HEQ dose (SD) & & & $11 ; 5.8(2.5)$ \\
\hline Olanzapine, $N_{;}$HEO dose (SD) & & & $17 ; 7.8(4.2)$ \\
\hline Quetiapine, N; HEQ dose (SD) & & & $5 ; 19.2(16.6)$ \\
\hline Clozapine, $N$; HEO dose (SD) & & & $8 ; 13.7(6.1)$ \\
\hline Aripiprazole, N; HEQ dose (SD) & & & $2 ; 9.0(4.2)$ \\
\hline Typical, $N$ & & & 5 \\
\hline Haloperidol, N; HEQ dose (SD) & & & $2 ; 3.5(0.7)$ \\
\hline Other typical9, N; HEQ dose (SD) & & & $3 ; 4.7(4.0)$ \\
\hline
\end{tabular}

${ }^{a}$ Data missing for three patients; ${ }^{b}$ Ranging from no education (0) to university (8). ' ${ }^{c}$ Estimated intelligence quotient (IQ). ${ }^{d}$ Other diagnoses include delusional disorder $(N=2)$, brief psychotic disorder ( $N=2)$, and psychosis not otherwise specified ( $N=3$ ). ${ }^{\circ}$ Positive and negative syndrome scale (PANSS), data missing for three patients; ${ }^{\dagger}$ Average haloperidol equivalent (HEQ) dose (mg); ${ }^{g}$ other typical medication includes flupentixol $(N=1)$, perfenazine $(N=1)$, and penfluridol ( $\left.N=1\right)$, data missing for five patients; * indicates a statistically significant difference (at $p<0.05)$.

Table 2 | Automated anatomical labeling regions per cerebellar ROI.

\begin{tabular}{|c|c|c|c|c|c|c|}
\hline $\begin{array}{l}\text { L anterior } \\
\text { hemisphere }\end{array}$ & $\begin{array}{l}\mathbf{R} \text { anterior } \\
\text { hemisphere }\end{array}$ & $\begin{array}{l}\text { Anterior } \\
\text { vermis }\end{array}$ & $\begin{array}{l}\text { L posterior } \\
\text { hemisphere }\end{array}$ & $\begin{array}{l}\text { R posterior } \\
\text { hemisphere }\end{array}$ & $\begin{array}{l}\text { Posterior } \\
\text { vermis }\end{array}$ & $\begin{array}{l}\text { Flocculo-nodular } \\
\text { lobe }\end{array}$ \\
\hline $\begin{array}{l}\text { L lobule III of } \\
\text { cerebellar } \\
\text { hemisphere }\end{array}$ & $\begin{array}{l}\text { R lobule III of } \\
\text { cerebellar } \\
\text { hemisphere }\end{array}$ & $\begin{array}{l}\text { Lobule I, II of } \\
\text { vermis }\end{array}$ & $\begin{array}{l}\text { L lobule VI of cerebellar } \\
\text { hemisphere }\end{array}$ & $\begin{array}{l}\mathrm{R} \text { lobule VI of cerebellar } \\
\text { hemisphere }\end{array}$ & $\begin{array}{l}\text { Lobule VI of } \\
\text { vermis }\end{array}$ & $\begin{array}{l}\text { L lobule } X \text { of cerebellar } \\
\text { hemisphere (flocculus) }\end{array}$ \\
\hline \multirow[t]{2}{*}{$\begin{array}{l}\text { L lobule IV, V of } \\
\text { cerebellar } \\
\text { hemisphere }\end{array}$} & $\begin{array}{l}\mathrm{R} \text { lobule IV, V of } \\
\text { cerebellar } \\
\text { hemisphere }\end{array}$ & $\begin{array}{l}\text { Lobule III of } \\
\text { vermis }\end{array}$ & $\begin{array}{l}\mathrm{L} \text { crus I of cerebellar } \\
\text { hemisphere }\end{array}$ & $\begin{array}{l}\mathrm{R} \text { crus I of cerebellar } \\
\text { hemisphere }\end{array}$ & $\begin{array}{l}\text { Lobule VII of } \\
\text { vermis }\end{array}$ & $\begin{array}{l}\mathrm{R} \text { lobule } \mathrm{X} \text { of cerebellar } \\
\text { hemisphere (flocculus) }\end{array}$ \\
\hline & & $\begin{array}{l}\text { Lobule IV, V of } \\
\text { vermis }\end{array}$ & $\begin{array}{l}\mathrm{L} \text { crus II of cerebellar } \\
\text { hemisphere } \\
\text { L lobule VIIB of } \\
\text { cerebellar hemisphere } \\
\text { L lobule VIII of } \\
\text { cerebellar hemisphere } \\
\text { L lobule IX of cerebellar } \\
\text { hemisphere }\end{array}$ & $\begin{array}{l}\text { R crus II of cerebellar } \\
\text { hemisphere } \\
\mathrm{R} \text { lobule VIIB of } \\
\text { cerebellar hemisphere } \\
\mathrm{R} \text { lobule VIII of } \\
\text { cerebellar hemisphere } \\
\text { R lobule IX of cerebellar } \\
\text { hemisphere }\end{array}$ & $\begin{array}{l}\text { Lobule VIII of } \\
\text { vermis } \\
\text { Lobule IX of } \\
\text { vermis }\end{array}$ & $\begin{array}{l}\text { Lobule } X \text { of vermis } \\
\text { (nodulus) }\end{array}$ \\
\hline
\end{tabular}

The 26 cerebellar regions distinguished by the automated anatomical labeling (AAL) atlas were assigned to one of seven cerebellar regions of interest; $L$, left; $R$, right. 


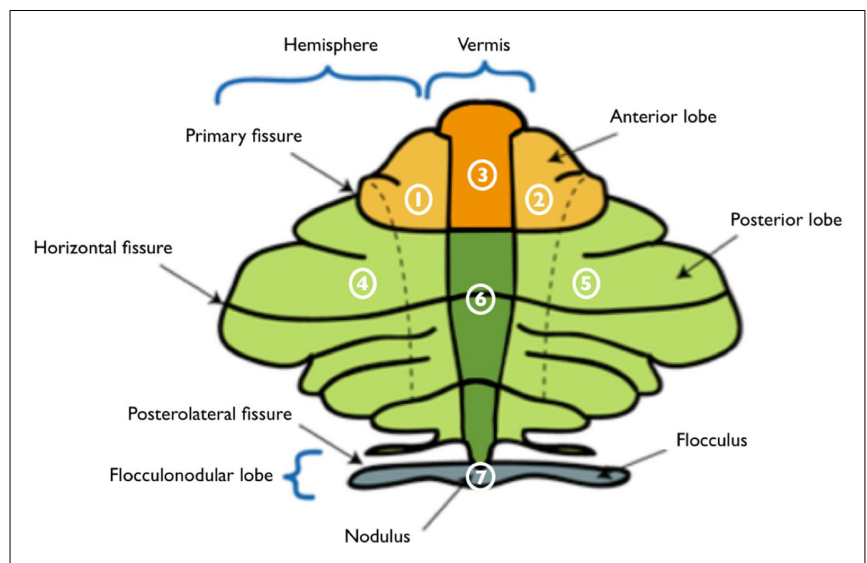

FIGURE 1 | Schematic illustration of the flattened cerebellar cortex and regions of interest. Major anatomical landmarks are depicted, as well as the cerebellar regions of interest, including the left (1) and right (2) anterior hemispheres, anterior vermis (3), left (4) and right (5) posterior

hemispheres, posterior vermis (6), and flocculo-nodular lobe (7). Adjusted to the image "Schematic representation of the major anatomical divisions of the cerebellum," source: en.wikipedia, released under the GNU Free Documentation License, version 1.3, 2008, published by the Free Software Foundation.

\section{FUNCTIONAL CONNECTIVITY ANALYSES}

Figure 2 illustrates the consecutive steps of the performed functional connectivity analysis. For each subject, 116 regional mean time-series were computed by averaging the voxel-based timeseries within each of the anatomically defined regions. Next, interregional correlation in resting-state time-series between each possible pair of the 26 cerebellar regions and the 90 cerebral regions in the AAL template was computed (Figure 2A). For each subject, functional connectivity per cerebellar ROI was then calculated by averaging the correlation coefficients of the AAL regions within each of the seven cerebellar regions (Figure 2B), rendering weighted correlation coefficients $r_{i, j}$ for the connections $\{i$, $j$ \} of each cerebellar ROI $i(N=7)$ with each cerebral region $j$ $(N=90)$. Interregional correlations were first computed and subsequently averaged for each ROI (rather than averaging at the level of regional time-series) as the ROIs may comprise more than one functional region and averaging distinct regional timeseries may lead signals to phase out when averaged. Fisher r-Z transforms were not applied as the correlations were normally distributed. Next, weighted correlation maps per subject group were computed for each cerebellar ROI (Figure 2C), rendering three matrices (for control, sibling, and patient groups) per ROI, with all 90 connections $\{i, j\}$ of the particular ROI $i$ on the $x$-axis and the number of subjects in the group $(N=41 ; 67 ; 62$ respectively) on the $y$-axis.

\section{HEALTHY CEREBELLAR FUNCTIONAL CONNECTIVITY}

In order to interpret possible differences in cerebellar functional connectivity between subject groups, the general pattern of functional connections per cerebellar ROI was investigated in the healthy control subjects. As functionally connected regions have been shown to exhibit a high degree of temporal coherence (Biswal et al., 1995; Van den Heuvel et al., 2008), higher interregional correlation in time-series was interpreted as a higher degree of functional connectivity between two regions.

\section{CEREBELLAR FUNCTIONAL CONNECTIVITY IN SCHIZOPHRENIA PATIENTS AND SIBLINGS}

Differences between subject groups in cerebellar functional connectivity patterns were examined using two functional connectivity measures. First, overall connectivity strength per cerebellar ROI was computed, providing information on the global level of communication between each cerebellar ROI and the rest of the brain.

\section{Analysis 1 overall connectivity strength $S$ per cerebellar ROI}

Connectivity strength $S_{i}$ of each cerebellar ROI i was computed as the average of all correlations between region $i$ and all extracerebellar regions $j$, providing information on the total level of connectivity of each cerebellar ROI (Figure 2D). Formally:

$\mathrm{S}_{\mathrm{i}}=\frac{\sum \mathrm{Fc}_{\mathrm{i}, \mathrm{j}}}{N_{\mathrm{j}}}$

Overall connectivity strength is a global measure of the extent to which the ROI is integrated in the brain network. However, it is not specific as to whether any particular connections are affected. Therefore, a pairwise approach was used next, to "zoom in" on discrete connections.

\section{Analysis 2 functional connectivity Fc of discrete connections}

Computed as the (averaged) correlation of each cerebellar region i with each extra-cerebellar region $j$ of the brain network, each functional connection $\mathrm{Fc}_{\mathrm{i}, \mathrm{j}}$ is an element of the set $\mathrm{Fc}_{\mathrm{i}}$ of all functional connections $(N=90)$ of the cerebellar ROI (Figure 2E):

$\mathrm{Fc}_{\mathrm{i}, \mathrm{j}} \in \mathrm{Fc}_{\mathrm{i}}$

In both analyses, the actual values (i.e., both positive and negative correlations) were used when averaging across correlation coefficients. As a consequence, negative correlations - which were both scarce and small in amplitude (all mean correlation coefficients $>-0.1)$ - were interpreted as lower levels of functional connectivity.

To exclude potential bias (in variance estimates) of family relationships within and between subject groups, the analyses were repeated with only unrelated individuals $(N=132)$, i.e., 46 schizophrenia patients, 47 siblings of patients, and 39 healthy control subjects.

\section{CLINICAL CORRELATES OF CEREBELLAR CONNECTIVITY}

Using linear regression analyses, the association between any possible changes in cerebellar connectivity measures and clinical variables (i.e., duration of illness; severity of PANSS symptoms and IQ) was investigated, and the dose of antipsychotic medication (in haloperidol equivalent) at the time of scanning was examined as a potential confounder.

\section{STATISTICAL ANALYSIS OF GROUP-DIFFERENCES}

Connectivity strength $S$ of each cerebellar ROI and functional connectivity Fc of each individual connection of schizophrenia 
A - Cerebellar AAL region $x$ - Cerebral AAL region y
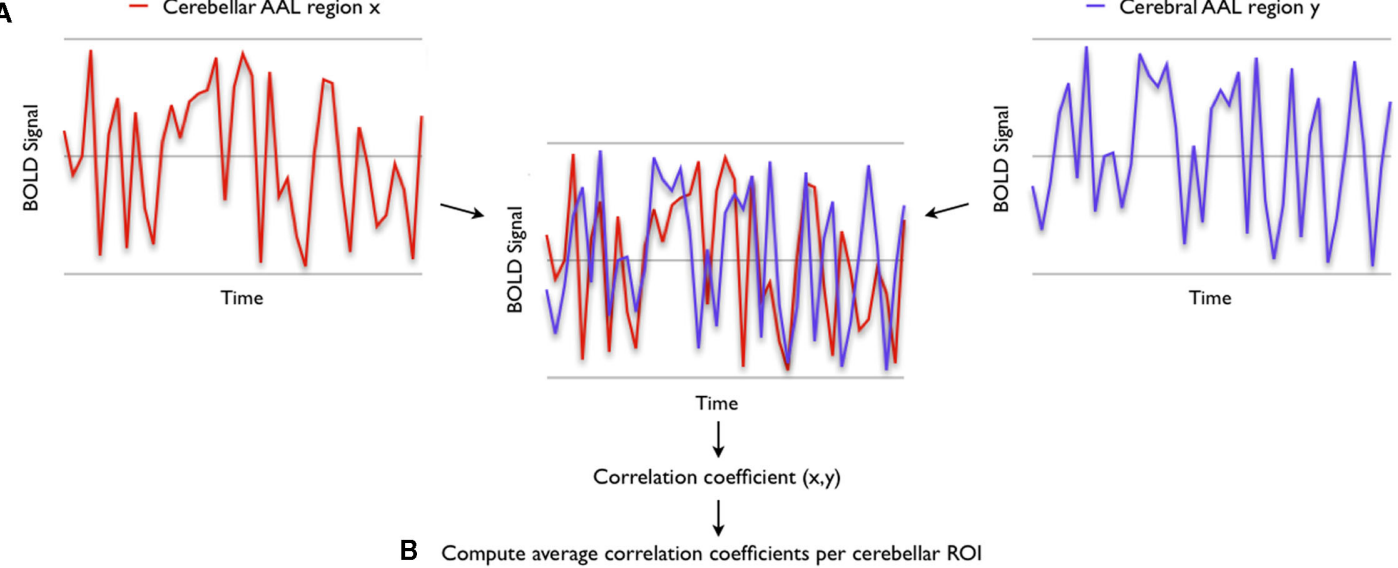

B

C

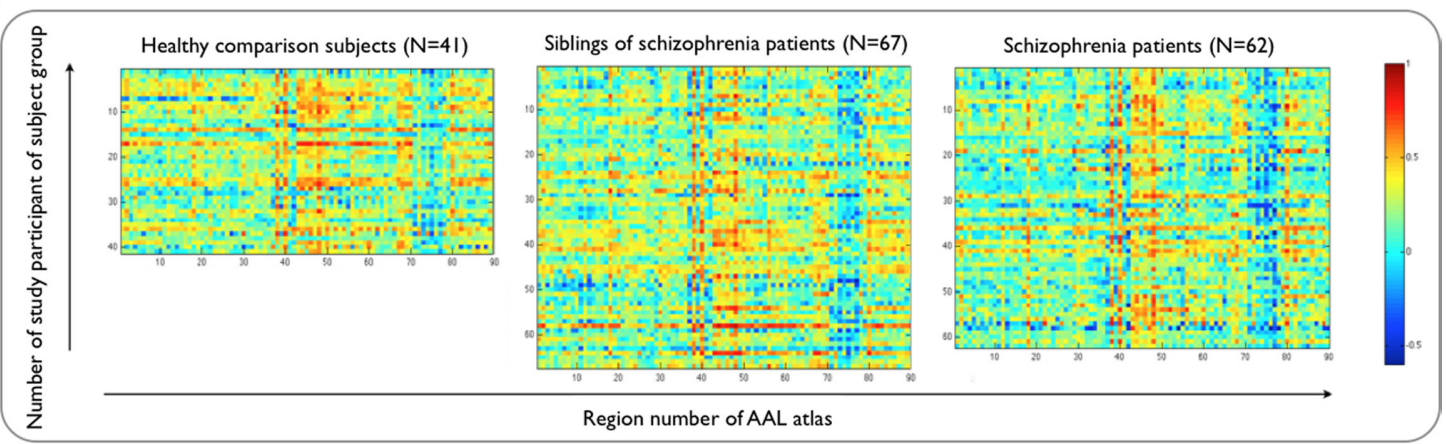

D Overall connectivity strength $S$ per cerebellar ROI
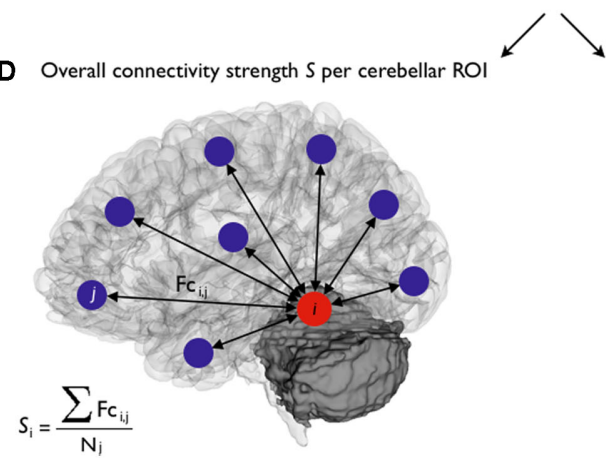

E Functional connectivity Fc of discrete connections

FIGURE 2 | Functional connectivity analysis. Consecutive steps of the functional connectivity analysis. (A) Computation of correlation between cerebellar and cerebral resting-state fMRI time-series, (B) calculation of correlation coefficients of cerebellar ROIs, (C) patients and siblings of schizophrenia patients were compared to those of the healthy comparison subjects. To examine the statistical significance of group-differences, permutation testing was used (5000 permutations; Bassett et al., 2008; Lynall et al., 2010; Van den Heuvel et al., 2010). To this end, random permutation of group assignment was performed, maintaining the original number of subjects per group, rendering three groups of 41, 62, and 67 randomly assigned subjects. Consequently, weighted correlation maps per randomly assigned subject group were computed for each cerebellar ROI. Using the resulting correlation maps, the connectivity measures were recalculated. This process was repeated 5000 times, resulting in a between-group difference null distribution of the connectivity measures. Finally, the observed differences between the original subject groups were compared to the normal distribution of differences after random permutation, to explore the null hypothesis that the observed differences were not determined by subject group membership. Finally, $p$-values were assigned to the group effects by computing (after random permutation) the percentage of findings that was more extreme than the observed difference between the original subject groups. In view of the number of tests performed in the connectivity strength analysis (Analysis 1, Figure 2D), a $p$-value of $<0.01$ was considered to 
indicate statistical significance, and a $p$-value of $>0.01$ and $<0.05$ to indicate a statistical trend. To account for the multiple comparisons of the second analysis (Analysis 2, Figure 2E), the effects on functional connectivity of discrete connections were subjected to a false discovery rate (FDR) threshold of $q=0.05$ (Benjami and Hochberg, 1995; Storey, 2002) over all functional connections $(N=90)$ of each cerebellar ROI, providing control over false positive findings.

\section{RESULTS}

\section{HEALTHY CEREBELLAR FUNCTIONAL CONNECTIVITY}

In the healthy comparison subjects, mean functional connectivity of the posterior hemispheres [mean $\mathrm{Fc}(\mathrm{SD})=0.27$ $(0.07)]>$ anterior hemispheres $[$ mean $\mathrm{Fc}(\mathrm{SD})=0.21(0.06)]$ and the right cerebellum $[$ mean $\mathrm{Fc}(\mathrm{SD})=0.27(0.08)]>$ left cerebellum [mean Fc $(\mathrm{SD})=0.22(0.06)]$ (both $p<0.0001)$. Furthermore, mean functional connectivity of the lateral cerebellum (i.e., hemispheres) [mean Fc $(\mathrm{SD})=0.24$ (0.06)] was significantly higher than that of midline structures (i.e., vermis and flocculo-nodular lobe) $[$ mean Fc $(\mathrm{SD})=0.14(0.05)](p<0.0001)$.

The highest levels of functional connectivity were found between the right anterior cerebellar hemisphere and right lingual gyrus (mean $\mathrm{Fc}=0.54$, SD 0.14), hippocampus [mean $\mathrm{Fc}(\mathrm{SD})=0.50(0.18)]$ and parahippocampal gyrus [mean Fc $(\mathrm{SD})=0.50(0.15)]$. Overall, cerebellar connections to posterior medial regions of the cerebral cortex [i.e., (pre)cuneus, calcarine sulcus, lingual gyrus] showed high levels of functional connectivity, irrespective of cerebellar ROI [mean Fc (SD) of all ROIs 0.26 (0.08), 0.26 (0.10); 0.34 (0.08), 0.32 (0.08); 0.32 (0.09), 0.29 (0.10); 0.29 (0.09), 0.33 (0.12); for the left and right cuneus; precuneus; calcarine sulcus; and lingual gyrus respectively]. The lowest levels of functional connectivity were observed for connections with the bilateral lentiform nucleus [mean Fc $(\mathrm{SD})=0.11(0.15)]$, in particular the globus pallidus [left; right mean Fc $(\mathrm{SD})=0.05(0.15) ; 0.09(0.15)]$, and with the gyrus rectus [left; right mean FC $(\mathrm{SD})=0.11(0.17) ; 0.09(0.17)$ ] (Figure 3).

\section{ANALYSIS 1 OVERALL CONNECTIVITY STRENGTH S OF CEREBELLAR ROIs}

Overall connectivity strength of the posterior and flocculonodular lobes of the cerebellum with the rest of the brain did not differ between groups, suggesting intact functional connectivity of posterior and flocculo-nodular cerebellar regions in schizophrenia. Connectivity strength of the right anterior hemisphere of the cerebellum however, was found to be $23 \%$ less $(p=0.01)$ in schizophrenia patients compared to healthy comparison subjects. Furthermore, on trend level, connectivity strength of the anterior vermis was less in both schizophrenia patients $(-27 \%, p=0.04)$ and siblings $(-22 \%, p=0.05)$, compared to healthy comparison subjects (Figure 4).

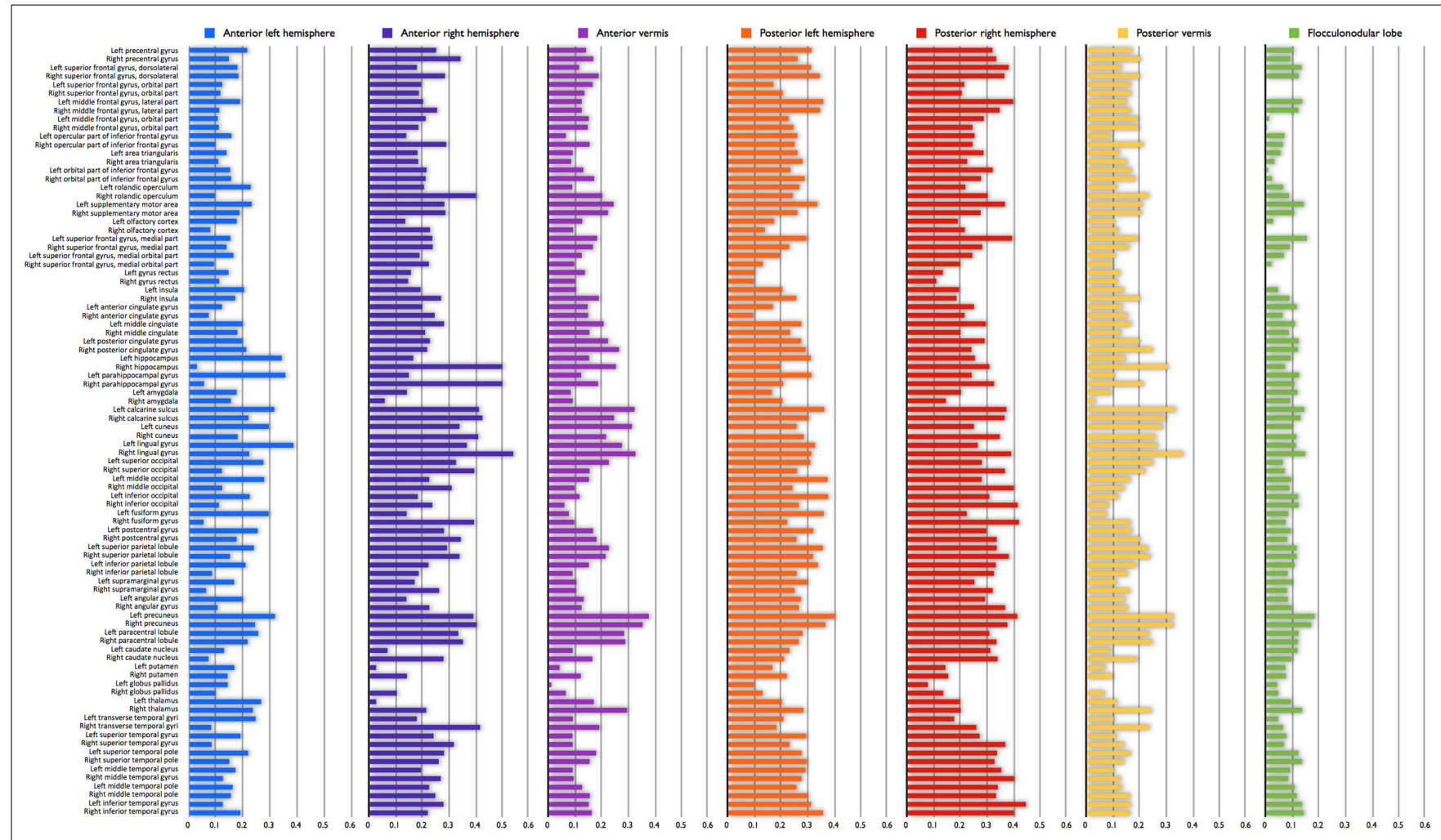

FIGURE 3 | Healthy cerebellar functional connectivity profile. Mean functional connectivity of all possible connections per cerebellar region of interest (ROI) in the healthy comparison sample. High correlations indicate high interregional functional connectivity; low correlations indicate low interregional functional connectivity. Negative correlations $(N=4$; mean (SD) [range] $=-0.05(0.18)[-0.07$ to -0.02$])$ are depicted as zero. 
ANALYSIS 2 FUNCTIONAL CONNECTIVITY OF DISCRETE CONNECTIONS: PATIENTS VERSUS CONTROLS

Impaired functional connectivity was most pronounced in the connections between the right anterior cerebellar hemisphere and vermis, and left-cerebral regions. Particularly between the right anterior vermis and the left-sided triangular part of the inferior frontal gyrus $(\mathrm{Fc}=-0.13, p=0.0024)$, supplementary motor area $(\mathrm{Fc}=-0.12, p=0.0014)$, middle cingulate gyrus $(\mathrm{Fc}=-12$, $p=0.0004)$, hippocampus $(\mathrm{Fc}=-0.17, p=0.0004)$, and thalamus $(\mathrm{Fc}=-0.16, p<0.0001)$. Functional connectivity between the latter two regions (i.e., left hippocampus and thalamus) and the anterior cerebellar vermis was also decreased in patients $(\mathrm{Fc}=-0.14, p=<0.0001 ; \mathrm{Fc}=-0.13, p=0.0008$ respectively). The only significant increase in functional connectivity in schizophrenia patients relative to controls was found between the posterior vermis and left lingual gyrus $(\mathrm{Fc}=0.15, p<0.0001)$. All these findings survived FDR-correction (Figure 5).

\section{ANALYSIS 2 FUNCTIONAL CONNECTIVITY OF DISCRETE CONNECTIONS: SIBLINGS VERSUS CONTROLS}

Compared to schizophrenia patients, the siblings demonstrated both similarities and disparities in functional connectivity of the
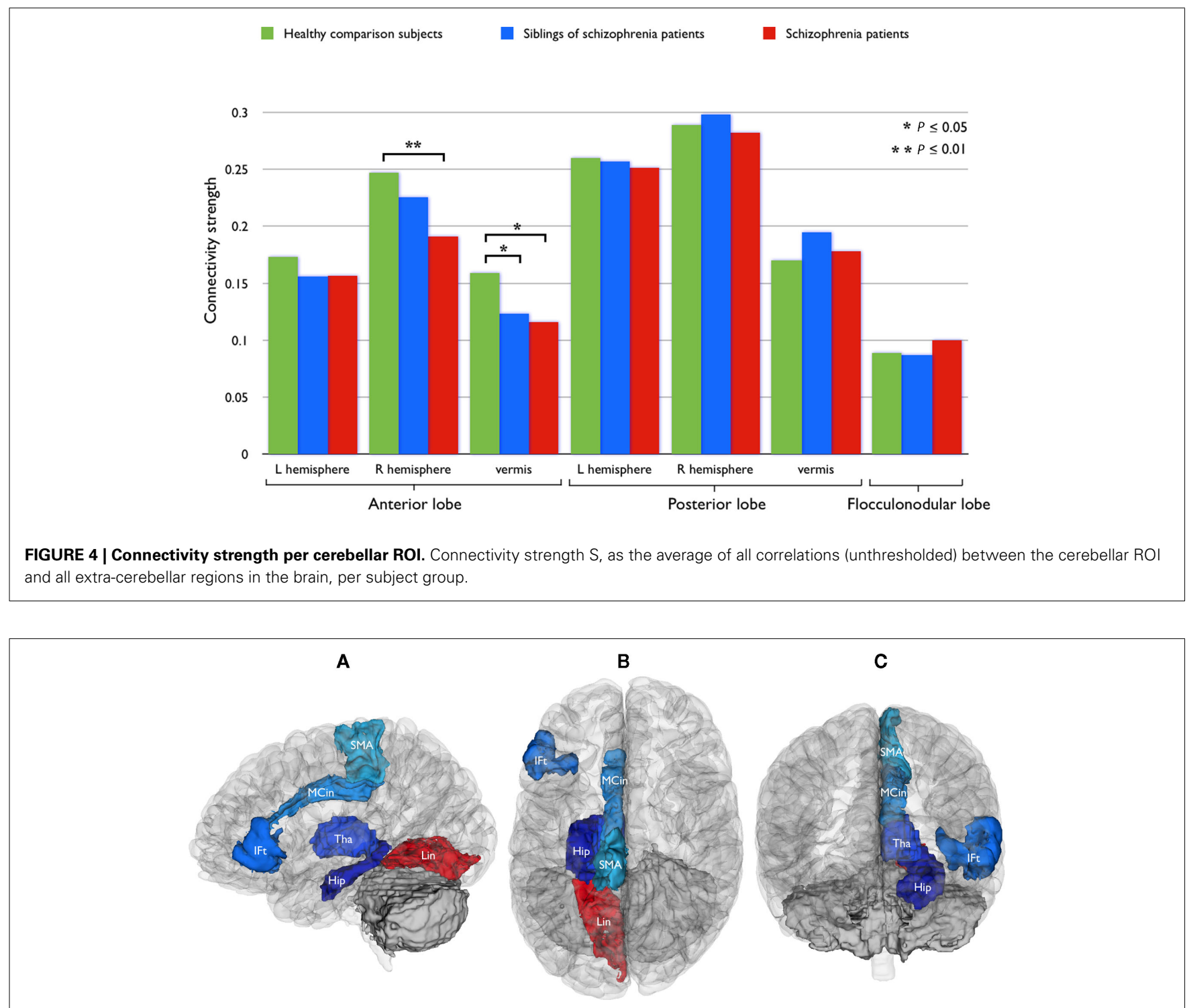

FIGURE 5 | Brain regions functionally dysconnected from the cerebellum in schizophrenia. Left lateral (A), superior (B), and anterior (C) views of the brain, showing the cerebral regions that were found to be functionally dysconnected (whole-brain significant) from the cerebellum. Blue signifies decreased functional connectivity with the cerebellum in schizophrenia patients compared to healthy comparison subjects (all $p<0.0025$, FDR-corrected), red indicates increased functional connectivity ( $p<0.0001$, FDR-corrected). Reduced functional connectivity involved the right anterior cerebellar hemisphere (IFt, inferior frontal gyrus, triangular part; SMA, supplementary motor area; MCin, Middle Cingulate gyrus; Hip, Hippocampus; Tha, Thalamus) and anterior vermis of the cerebellum (Hip, Hippocampus; Tha, Thalamus). Increased functional connectivity involved the posterior cerebellar vermis (Lin, Lingual gyrus). 
cerebellum. Siblings showed decreased functional connectivity (compared to control subjects) between the anterior cerebellar vermis and the triangular part of the left inferior frontal gyrus $(\mathrm{Fc}=-0.08, p=0.009)$, left insula $(\mathrm{Fc}=-0.08, p=0.007)$, and left hippocampus $(\mathrm{Fc}=-0.11, p=0.002)$, as well as between the right anterior cerebellar hemisphere and left hippocampus $(\mathrm{Fc}=-0.12, p=0.003)$, resembling the findings in patients. However, these findings were not as strong as in patients, as they failed to reach whole-brain significance (note that impaired functional connectivity between the anterior cerebellar vermis and left insula did not reach whole-brain significance in both siblings and patients), and should be considered exploratory. Conversely, functional connectivity to other brain regions that were found to be dysconnected from the cerebellum in schizophrenia patients (e.g., left thalamus, middle cingulate gyrus, and supplementary motor area) was not decreased in siblings of schizophrenia patients, compared to the healthy control subjects. Table 3 depicts all findings at $p<0.01$ in schizophrenia patients and siblings of patients.

Importantly, as siblings were psychiatrically healthy (see inclusion criteria and SIS-R scores, Table 1 ) and, on average, around 30 years of age, it is unlikely that any would still convert to illness. Lastly, repeating the functional connectivity analyses in a reduced

Table 3 | Absolute differences in functional connectivity between subject groups.

\begin{tabular}{|c|c|c|c|c|c|c|c|c|c|c|c|c|c|c|}
\hline & \multicolumn{14}{|c|}{ Cerebellar regions } \\
\hline & \multicolumn{2}{|c|}{$\begin{array}{l}\text { L anterior } \\
\text { hemisphere }\end{array}$} & \multicolumn{2}{|c|}{$\begin{array}{l}\text { R anterior } \\
\text { hemisphere }\end{array}$} & \multicolumn{2}{|c|}{$\begin{array}{l}\text { Anterior } \\
\text { vermis }\end{array}$} & \multicolumn{2}{|c|}{$\begin{array}{l}\text { L posterior } \\
\text { hemisphere }\end{array}$} & \multicolumn{2}{|c|}{$\begin{array}{l}\text { R posterior } \\
\text { hemisphere }\end{array}$} & \multicolumn{2}{|c|}{$\begin{array}{l}\text { Posterior } \\
\text { vermis }\end{array}$} & \multicolumn{2}{|c|}{$\begin{array}{l}\text { Flocculo-nodular } \\
\text { lobe }\end{array}$} \\
\hline & Sz & Sib & Sz & Sib & $\mathbf{S z}$ & Sib & $\mathbf{S z}$ & Sib & Sz & Sib & $\mathrm{Sz}$ & Sib & Sz & Sib \\
\hline \multicolumn{15}{|l|}{ CEREBRAL REGIONS } \\
\hline $\begin{array}{l}\text { R superior frontal gyrus, } \\
\text { dorsolateral }\end{array}$ & & & 0.10 & & & & & & & & & & & \\
\hline $\begin{array}{l}\text { L superior frontal gyrus, } \\
\text { orbital }\end{array}$ & & & 0.11 & & & 0.07 & & & & & & & & \\
\hline L middle frontal gyrus, orbital & & & 0.10 & & & & & & & & & & & \\
\hline $\begin{array}{l}\mathrm{L} \text { inferior frontal gyrus, } \\
\text { triangular }\end{array}$ & & & $0.13^{*}$ & & 0.09 & 0.08 & & & & & & & & \\
\hline L supplementary motor area & & & $0.12^{*}$ & & 0.09 & & & & & & & & & \\
\hline R supplementary motor area & & & 0.10 & & & & & & & & & & & \\
\hline $\begin{array}{l}\text { R superior frontal gyrus, } \\
\text { medial }\end{array}$ & & & 0.09 & & & & & & & & & & & \\
\hline $\begin{array}{l}\text { L superior frontal gyrus, } \\
\text { medialorbital }\end{array}$ & 0.08 & & & & & & & & & & & & & \\
\hline L gyrus rectus & 0.09 & & & & & & & & & & & & & \\
\hline $\mathrm{R}$ gyrus rectus & & & & & & 0.07 & & & & & & & & \\
\hline $\mathrm{L}$ insula & & & & & 0.10 & 0.08 & & & & & & & & \\
\hline $\mathrm{L}$ anterior cingulate gyrus & & & & & & & & & & & & 0.07 & & \\
\hline L middle cingulate gyrus & & & $0.12^{*}$ & & 0.10 & & & & & & & & & \\
\hline L hippocampus & & & $0.17^{*}$ & 0.12 & $0.14^{*}$ & 0.11 & & & & & 0.11 & & & \\
\hline$L$ lingual gyrus & & & & & & & & & & & $0.15^{*}$ & & 0.08 & \\
\hline $\mathrm{R}$ lingual gyrus & & & & & & & & & & & 0.10 & & & \\
\hline R inferior occipital gyrus & & & 0.11 & & & & & & & & & & & \\
\hline L inferior parietal lobule & & & & & & 0.08 & & & & & & & & \\
\hline L paracentral lobule & & & 0.10 & & & & & & & & & & & \\
\hline L caudate nucleus & & & & & & & & & & & & 0.08 & & \\
\hline R caudate nucleus & & & & & 0.09 & & & & & & & & & \\
\hline R globus pallidus & & & 0.10 & & & & & & & & & & & \\
\hline $\mathrm{L}$ thalamus & & & $0.16^{*}$ & & $0.13^{*}$ & & & & & & 0.11 & & & \\
\hline L superior temporal gyrus & & & & & & & & & & & & & 0.06 & \\
\hline R superior temporal pole & & & 0.11 & & & & & & & & & & & \\
\hline
\end{tabular}

Absolute difference in interregional correlation coefficients between schizophrenia patients and healthy comparison subjects; and siblings of patients and healthy comparison subjects. Decreased functional connectivity relative to healthy comparison subjects is displayed in blue, increased functional connectivity in red. Findings at $p<0.01$ are depicted; * indicates whole-brain significance after FDR-correction. Sz, schizophrenia patients; Sib, siblings of schizophrenia patients, L, left-sided, R, right-sided. 
number of study participants that were completely unrelated did not alter the findings.

\section{CLINICAL CORRELATES OF CEREBELLAR CONNECTIVITY}

Cerebellar functional connectivity measures that were significantly different in schizophrenia patients, compared to healthy comparison subjects, were investigated for associations with medication dose, duration of illness, symptom severity, and global cognitive performance, using linear regression analysis. No association between the dose of antipsychotic medication at the time of scanning and cerebellar functional connectivity was found (all $p>0.420$ ). After multiple comparison correction, there were no significant associations between aberrant cerebellar functional connectivity measures and clinical variables. On trend level, a counter-intuitive association between more negative symptoms and increased Fc between the cerebellar right anterior hemisphere and left hippocampus was observed (Table 4).

\section{DISCUSSION}

The main finding of our study is the presence of an aberrant level of functional connectivity of the cerebellum in schizophrenia patients and their healthy siblings. Our findings suggest that in schizophrenia, the cerebellum, specifically the vermis, and right anterior hemisphere, is functionally dysconnected from a range of left-cerebral cortical and subcortical regions, including frontal, cingulate, and occipital regions, as well as the thalamus and hippocampus. Importantly, unaffected siblings of schizophrenia patients demonstrated several similarities in cerebellar functional dysconnectivity compared to patients, in particular reduced functional connectivity of the cerebellum to the left hippocampus. This overlap suggests that cerebellar dysconnectivity may be related, at least in part, to familial (and possibly genetic) factors.

Disparities in cerebellar connectivity between schizophrenia patients and their siblings were also found. Of these, the dissimilarity in cerebellar-thalamic dysconnectivity may be the most meaningful, as the thalamus is the obligatory relay for all efferent cerebellar projections to the cortex. In this context, our findings may imply that cerebellar-thalamic dysconnectivity might be related more to the manifestation of the illness, than to familial (or genetic) factors.

Although the term "dysconnectivity" emphasizes the notion of abnormal, rather than necessarily decreased, functional integration between brain regions (Stephan et al., 2009a), structural and functional connectivity studies in schizophrenia have mostly reported reduced (rather than increased) connectivity in schizophrenia (Petterson-Yeo et al., 2011). In line with these findings, our study shows that in schizophrenia, functional connectivity of the cerebellum is mostly decreased. Furthermore, studies have reported changing patterns of reduced connectivity across the different stages of disease, from chronic schizophrenia to individuals at high (genetic) risk for psychosis (including healthy first-degree relatives of schizophrenia patients; PettersonYeo et al., 2011). These findings support the presently reported overlap in cerebellar dysconnectivity between patients and siblings. Additional support for this finding comes from a recent study examining functional connectivity within and between four predefined functional networks: the default mode network and three cognitive control (frontal-parietal, cingulo-opercular, and cerebellar) networks (Repovs et al., 2011). Compared to controls, reduced connectivity between cerebellar, frontal-parietal, and cingulo-opercular networks was shown in both schizophrenia patients and siblings of patients. Intriguingly, greater reductions in connectivity between the frontal-parietal and cerebellar regions were found to be robustly predictive of worse cognitive performance across groups and predictive of more disorganization symptoms among patients. The association between cognitive performance and cerebellar functional connectivity is not replicated by the present study. However, as modest cognitive deficits are reported in adult patients with (especially right-sided) cerebellar lesions, while similar lesions in children lead to pervasive impairments (suggesting a critical role for the cerebellum in development; Alexander et al., 2011), the relationship between cerebellar abnormalities and cognition may not be straightforward and may depend on factors such as age of illness onset. Regarding the relationship with clinical symptoms, the meaning of the counter-intuitive trend between greater severity of PANSS

Table 4 | Clinical correlates of aberrant functional connectivity measures.

\begin{tabular}{|c|c|c|c|c|c|c|c|c|c|c|}
\hline & \multicolumn{10}{|c|}{ Aberrant functional connectivity measures } \\
\hline & $\begin{array}{l}\text { S } \\
\text { Cer2 }\end{array}$ & $\begin{array}{l}\text { S } \\
\text { Cer3 }\end{array}$ & $\begin{array}{l}\text { Fc } \\
\text { Cer2-IFt }\end{array}$ & $\begin{array}{l}\text { Fc } \\
\text { Cer2-SMA }\end{array}$ & $\begin{array}{l}\text { Fc } \\
\text { Cer2-MCin }\end{array}$ & $\begin{array}{l}\text { Fc } \\
\text { Cer2- Hip }\end{array}$ & $\begin{array}{l}\text { Fc } \\
\text { Cer2-Tha }\end{array}$ & $\begin{array}{l}\text { Fc } \\
\text { Cer3-Hip }\end{array}$ & $\begin{array}{l}\text { Fc } \\
\text { Cer3-Tha }\end{array}$ & $\begin{array}{l}\text { Fc } \\
\text { Cer6-Lin }\end{array}$ \\
\hline Duration of illness & -0.21 & -0.07 & -0.29 & -0.26 & -0.15 & -0.05 & -0.10 & 0.10 & 0.12 & -0.22 \\
\hline \multicolumn{11}{|l|}{ PANSS } \\
\hline Total score & 0.24 & 0.12 & 0.09 & 0.02 & 0.29 & 0.33 & 0.31 & 0.26 & 0.10 & -0.10 \\
\hline Positive symptoms & 0.16 & 0.15 & -0.01 & 0.01 & 0.23 & 0.17 & 0.26 & 0.16 & 0.03 & -0.01 \\
\hline Negative symptoms & 0.17 & 0.07 & 0.05 & -0.01 & 0.22 & $0.37^{*}$ & 0.27 & 0.25 & 0.18 & -0.07 \\
\hline 10 & -0.02 & -0.04 & -0.15 & -0.02 & -0.03 & -0.29 & -0.16 & -0.11 & -0.07 & -0.12 \\
\hline
\end{tabular}

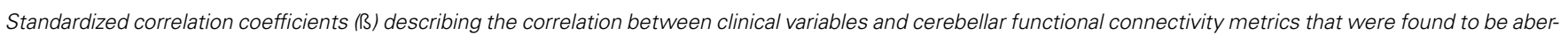

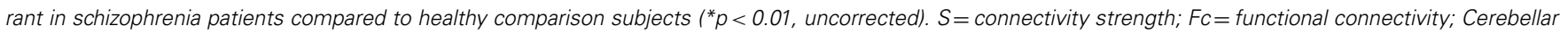

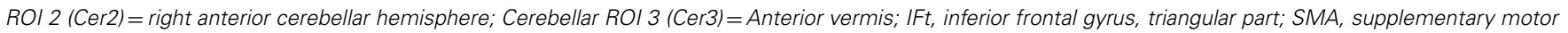
area, MCin, middle cingulate gyrus, Hip, hippocampus, Tha, thalamus, Lin, lingual gyrus. 
negative symptoms and increased functional connectivity between the cerebellar anterior right hemisphere and left hippocampus is unclear, but may be an indication of discrete symptom dimensions in schizophrenia with distinct underlying neurobiology (Ke et al., 2010).

Our findings suggest that functional dysconnectivity of the cerebellum to the cerebrum involves mainly anterior and vermal areas of the cerebellum. The vermis has previously been labeled the "limbic cerebellum," as the (anterior) vermis is the principal cerebellar target of limbic projections (Schmahmann, 2000). Furthermore, behavioral studies support a relationship between cerebellar midline structures and the modulation of emotion (Heath and Harper, 1974; Stoodley and Schmahmann, 2010), and lesions of the vermis have been shown to produce affective symptoms, ranging from emotional blunting and depression to disinhibition and psychotic features (Schmahmann and Sherman, 1998). The observed reduction in functional integration between this cerebellar region and limbic regions such as the hippocampus, cingulate cortex and (anterior nuclei of) the thalamus, could perhaps be interpreted in this context. Notably, the present study found no evidence for impaired functional connectivity of the posterior cerebellar hemispheres, while these cerebellar regions have been preferentially linked to cognitive processing (Habas et al., 2009; Krienen and Buckner, 2009). Cerebellar involvement in schizophrenia may be either limited to the vermis and anterior lobe, or involvement of the posterior cerebellar hemispheres in (cognitive deficits in) schizophrenia may be more subtle, attenuating in our whole-brain approach with corresponding stringent correction for multiple comparisons. Alternatively, as a distinct topographic organization of the cerebellum has been proposed in which certain areas of the cerebellar cortex interact specifically with certain areas of the cerebral cortex (Stoodley and Schmahmann, 2010; Buckner et al., 2011), the relatively large posterior hemispheres (compared to the other cerebellar ROIs) may have such distributed functional connections, that "picking up" on dysconnectivity of any particular connection(s) was precluded by our definition of ROIs.

The neuronal basis for dysconnectivity remains to be established. It could result from either aberrant wiring of connections during development or from impaired synaptic plasticity (or both; Stephan et al., 2009a). As studies have suggested a link between functional and structural brain connectivity (Hagmann et al., 2008; Honey et al., 2009; Van den Heuvel et al., 2009), our findings may reflect impaired structural connections (i.e., white matter tracts) between affected brain regions. Indeed, reduced fractional anisotropy (FA), commonly interpreted as reduced integrity, of cerebellar white matter tracts has been shown in schizophrenia (Kanaan et al., 2009; Kyriakopoulos and Frangou, 2009). Accompanied by normal mean diffusivity, this FA reduction is most likely due to disordered microstructural architecture, rather than disordered myelination (Kanaan et al., 2009). Accordingly, one study using magnetic resonance spectroscopic imaging reported decreased levels of a putative neuronal/axonal marker in the anterior cerebellar vermis, suggesting dysfunction or loss of neurons in that region (Deicken et al., 2001). Cerebellar dysconnectivity may thus result from decreased neurons or disordered neuronal architecture in the cerebellum.
Some issues have to be taken into account when interpreting the results of our study. First, medicated patients were studied and antipsychotic mediation has been shown to affect cerebellar functional connectivity (Stephan et al., 2009b). However, the haloperidol equivalent dose of antipsychotic medication at the time of scanning was not associated with functional connectivity measures and the unmedicated siblings of schizophrenia patients showed similar abnormalities in cerebellar functional connectivity, suggesting that cerebellar dysconnectivity is unlikely to be due to antipsychotic medication alone. Second, there was a preponderance of men in the schizophrenia patients, which was not paralleled in the healthy control and sibling groups. Nonetheless, as we applied permutation testing, it is very unlikely that our findings were driven by any other factor than group membership (i.e., patient, sibling, or control), as group-differences determined by other factors - such as gender - would not have been among the most extreme findings after random permutation of group assignment and thus not be deemed significant. Furthermore, our subdivision of the cerebellum may not be the optimal division of the cerebellum in terms of its functional connections. As the connectional properties of the cerebellum (i.e., connected to the cerebral cortex only by ways of polysynaptic connections) are relatively unamenable to traditional anatomical methods, the connectional topography of the cerebellum remains largely unmapped (O'Reilly et al., 2010). Recent studies using transneuronal tracing techniques and functional neuroimaging have provided some insight into the functional organization of the cerebellar cortex, but both distinct and overlapping functional zones of the cerebellar cortex have been reported and findings diverge between studies (O'Reilly et al., 2010; Stoodley and Schmahmann, 2010; Buckner et al., 2011). It was therefore decided to adhere to a broad division of the cerebellum, based on gross anatomy. Moreover, although various preprocessing steps were used to deal with potential confounds associated with resting-state fMRI (e.g., cardiorespiratory oscillations, head movement, scanner noise), influences of these factors cannot be ruled out. There is also an inherent risk of potential bias associated with cortical parcellation methods such as the AAL template, as the spatial scale of nodal parcellation has been shown to influence brain network properties (Wang et al., 2009; Fornito et al., 2010). New approaches are being developed to perform whole-brain connectivity mapping using individual pairs of voxels, without the need of arbitrary parcellation of the cortex (Zalesky et al., 2010), but interpreting voxels as distinct information processing units has its own limitations and inevitably, investigations of in vivo wholebrain functional connectivity have a resolution limit (Wig et al., 2011). Finally, although we interpret our findings of cerebellar dysconnectivity in schizophrenia in terms of underlying neurobiology and psychopathology, it should be noted that cause and effect cannot be derived from our cross-sectional data.

In conclusion, our study indicates that the cerebellum, in particular the vermis and right anterior hemisphere, is functionally dysconnected in schizophrenia. Furthermore, the observed overlap in cerebellar dysconnectivity between schizophrenia patients and their healthy siblings suggest that cerebellar dysconnectivity is related, at least in part, to familial risk for psychosis. Whether, and if so to what extent, cerebellar dysconnectivity is mediated by genetic factors needs to be established in genetic studies. 


\section{REFERENCES}

Alexander, M. P., Gillingham, S., Schweizer, T., and Stuss, D. T. (2011). Cognitive impairments due to focal cerebellar injuries in adults. Cortex. doi:10.1016/j.cortex.2011.03.012. [Epub ahead of print].

American Psychiatric Association. (1994). Diagnostic and Statistical Manual of Mental Disorders, 4th Edn. Washington: American Psychiatric Association.

Andreasen, N. C., Flaum, M., and Arndt, S. (1992). The comprehensive assessment of symptoms and history (CASH): an instrument for assessing diagnosis and psychopathology. Arch. Gen. Psychiatry 49, 615-623.

Andreasen, N. C., Paradiso, S., and O'Leary, D. S. (1998). "Cognitive dysmetria" as an integrative theory of schizophrenia: a dysfunction in cortical-subcorticalcerebellar circuitry? Schizophr. Bull. 24, 203-218.

Andreasen, N. C., and Pierson, R. (2008). The role of the cerebellum in schizophrenia. Biol. Psychiatry 64, 81-88.

Assaf, Y., and Pasternak, O. (2008). Diffusion tensor imaging (DTI)based white matter mapping in brain research: a review. J. Mol. Neurosci. 34, 51-61.

Bassett, D. S., and Bullmore, E. T. (2009). Human brain networks in health and disease. Curr. Opin. Neurol. 22, 340-347.

Bassett, D. S., Bullmore, E. T., Verchinski, B. A., Mattay, V. S., Weinberger, D. R., and Meyer-Linderberg, A. (2008). Hierarchical organization of human cortical networks in health and schizophrenia. J. Neurosci. 28, 9239-9248.

Becerril, K. E., Repovs, G., and Barch, D. M. (2011). Error processing network dynamics in schizophrenia. Neuroimage 54, 1495-1505.

Benjami, Y., and Hochberg, Y. (1995). Controlling for the false discovery rate: a practical and powerful approach to multiple testing. $J$. $R$. Stat. Soc. Series B Stat. Methodol. 57, 289-300.

Biswal, B., Van Kylen, J., and Hyde, J. S. (1997). Simultaneous assessment of flow and BOLD signals in resting state functional connectivity maps. NMR. Biomed. 10, 165-170.

Biswal, B., Yetkin, F. Z., Haughton, V. M., and Hyde, J. S. (1995). Functional connectivity in the motor cortex of resting human brain using echoplanar MRI. Magn. Reson. Med. 34, 537-541.

Bleuler, E. (1911). Dementia Praecox or the Group of Schizophrenias (In
German). New York, NY: International Universities Press.

Buckner, R. L., Krienen, F. M., Castellanos, A., Diaz, J. C., and Yeo, B. T. (2011). The organization of the human cerebellum estimated by intrinsic functional connectivity. $J$. Neurophysiol. 106, 2322-2345.

Deicken, R. F., Feiwell, R., Schuff, N., and Soher, B. (2001). Evidence for altered cerebellar vermis neuronal integrity in schizophrenia. Psychiatry Res. 107, 125-134.

Fornito, A., Yoon, J., Zalesky, A., Bullmore, E. T., and Carter, C. S. (2011). General and specific functional connectivity disturbances in first-episode schizophrenia during cognitive control performance. Biol. Psychiatry 70, 64-72.

Fornito, A., Zalesky, A., and Bullmore, E. T. (2010). Network scaling effects in graph analytic studies of human resting-state FMRI data. Front. Syst. Neurosci. 4:22. doi:10.3389/fnsys.2010.00022

Friston, K. J. (1998). The disconnection hypothesis. Schizophr. Res. 30, 115-125.

Friston, K. J., Frith, C. D., Liddle, P. F., and Frackowiak, S. J. (1993). Functional connectivity: the principalcomponent analysis of large (PET) data sets. J. Cereb. Blood Flow Metab. 13, 5-14.

Habas, C., Kamdar, N., Nguyen, D., Prater, K., Beckmann, C. F., Menon, V., and Greicus, M. D. (2009). Distinct cerebellar contributions to intrinsic connectivity networks. J. Neurosci. 29, 8586-8594.

Hagmann, P., Cammoun, L., Gigandet, X., Meuli, R., Honey, C. J., Wedeen, V. J., and Sporns, O. (2008). Mapping the structural core of human cerebral cortex. PLoS Biol. 6, e159. doi:10.1371/journal.pbio.0060159

Heath, R. G., and Harper, J. W. (1974). Ascending projections of the cerebellar fastigial nucleus to the hippocampus, amygdala, and other temporal lobe sites: evoked potential and histological studies in monkeys and cats. Exp. Neurol. 45, 2682-2687.

Honey, C. J., Sporns, O., Cammoun, L., Gigandet, X., Thiran, J. P., Meuli, R., and Hagmann, P. (2009). Predicting human resting-state functional connectivity from structural connectivity. Proc. Natl. Acad. Sci. U.S.A. 106, 2035-2040.

Honey, G. D., Pomarol-Clotet, E., Corlett, P. R., Honey, R. A. E., McKenna, P. J., Bullmore, E. T., and Flechter, P. C. (2005). Functional dysconnectivity in schizophrenia associated with attentional modulation of motor function. Brain 128, 2597-2611.

Kanaan, R. A. A., Borgwardt, S., McGuire, P. K., Craig, M. C.,
Murphy, D. G. M., Picchioni, M., Shergill, S. S., Jones, D. K., and Catani, M. (2009). Microstructural organization of cerebellar tracts in schizophrenia. Biol. Psychiatry 66, 1067-1069.

Kandel, E. R., Schwartz, J. H., and Jessell, T. M. (2000). "The cerebellum," in Principles of Neural Science, 4th Edn, eds J. Butler, and H. Lebowitz (New York, NY: McGraw-Hill Companies), 833-835.

Kay, S. R., Fiszbein, A., and Opler, L. A. (1987). The positive and negative syndrome scale (PANSS) for schizophrenia. Schizophr. Bull. 13, 261-276.

Ke, M., Zou, R., Shen, H., Huang, X., Zhou, Z., Liu, Z., Xue, Z., and Hu, D. (2010). Bilateral functional asymmetry disparity in positive and negative schizophrenia revealed by resting-state fMRI. Psychiatry Res. 182, 30-39.

Kendler, K. S., Lieberman, J. A., and Walsh, D. (1989). The Structured Interview for Schizotypy (SIS): a preliminary report. Schizophr. Bull. 15, 559-571.

Kim, D., Burge, J., Lane, T., Pearlson, G. D., Kiehl, K. A., and Calhoun, V. D. (2008). Hybrid ICABayesian network approach reveals distinct effective connectivity differences in schizophrenia. Neuroimage 42, 1560-1568.

Kraepelin, E. (1919). Dementia Praecox and Paraphrenia. Edinburgh: Livingstone.

Krienen, F. M., and Buckner, R. L. (2009). Segregated fronto-cerebellar circuits revealed by intrinsic functional connectivity. Cereb. Cortex 19, 2485-2497.

Kroken, R. A., Johnsen, E., Ruud, T., Wentzel-Larsen, T., and Jørgensen, H. A. (2009). Treatment of schizophrenia with antipsychotics in Norwegian emergency wards, a cross-sectional national study. BMC Psychiatry 9, 24. doi:10.1186/1471-244X-9-24

Kyriakopoulos, M., and Frangou, S. (2009). Recent diffusion tensor imaging findings in early stages of schizophrenia. Curr. Opin. Psychiatry 22, 168-176.

Lynall, M. E., Bassett, D. S., Kerwin, R., McKenna, P. J., Kitzbichler, M., Muller, U., and Bullmore, E. T. (2010). Functional connectivity and brain networks in schizophrenia. $J$. Neurosci. 30, 9477-9487.

O’Reilly, J. X., Beckmann, C. F., Tomassini, V., Ramnane, N., and Johansen-Berg, H. (2010). Distinct and overlapping functional zones in the cerebellum defined by restingstate functional connectivity. Cereb. Cortex 20, 953-965.
Petterson-Yeo, W., Allen, P., Benetti, S., McGuire, P., and Mechelli, A. (2011). Dysconnectivity in schizophrenia: where are we now? $\mathrm{Neu}$ rosci. Biobehav. Rev. 35, 1110-1124.

Ramsey, N. F., Kirkby, B. S., Van Gelderen, P., Berman, K. F., Duyn, J. H., Frank, J. A., Mattay, V. S., Van Horn, J. D., Esposito, G., Moonen, C. T., and Weinberger, D. R. (1996). Functional mapping of human sensorimotor cortex with 3D BOLD fMRI correlates highly with $\mathrm{H} 2(15) \mathrm{O}$ PET rCBF. J. Cereb. Blood Flow Metab. 16, 755-764.

Ramsey, N. F., Van den Heuvel, M. P., Kho, K. H., and Leijten, F. S. (2006). Towards human BCI applications based on cognitive brain systems: an investigation of neural signals recorded from the dorsolateral prefrontal cortex. IEEE Trans. Neural Syst. Rehabil. Eng. 14, 214-217.

Repovs, G., Csernansky, J. G., and Barch, D. M. (2011). Brain network connectivity in individuals with schizophrenia and their siblings. Biol. Psychiatry 69, 967-973.

Rubinov, M., and Bassett, D. S. (2011). Emerging evidence of connectomic abnormalities in schizophrenia. J. Neurosci. 31, 6263-6265.

Schmahmann, J. D. (1998). Dysmetria of thought: clinical consequences of cerebellar dysfunction on cognition and affect. Trends Cogn. Sci. (Regul. Ed.) 2, 362-371.

Schmahmann, J. D. (2000). The role of the cerebellum in affect and psychosis. J. Neurolinguistics 13, 189-214.

Schmahmann, J. D., and Caplan, D. (2006). Cognition, emotion and the cerebellum. Brain 129, 290-292.

Schmahmann, J. D., and Sherman, J. C. (1998). The cerebellar cognitive affective syndrome. Brain 121, 561-579.

Stephan, K. E., Friston, K. J., and Frith, C. D. (2009a). Dysconnection in schizophrenia: from abnormal synaptic plasticity to failures of self-monitoring. Schizophr. Bull. 35, 509-527.

Stephan, K. E., Magnotta, V. A., White, T., Arndt, S., Flaum, M., O'Leary, D. S., and Andreasen, N. C. (2009b). Effects of olanzapine on cerebellar functional connectivity in schizophrenia measured by fMRI during a simple motor task. Psychol. Med. 31, 1065-1078.

Stinissen, J., Willems, P. J., Coetsier, P., and Hulsman, W. L. L. (1970). Manual of the Dutch Translation of the Wechsler Adult Intelligence Scale. Amsterdam: Swets. 
Stoodley, C. S., and Schmahmann, J. D. (2010). Evidence for topographic organization in the cerebellum of motor control versus cognitive and affective processing. Cortex $46,831-844$

Storey, J. D. (2002). A direct approach to false discovery rates. J. R. Stat. Soc. Series B Stat. Methodol. 64, 479-498.

Sullivan, P. F., Kendler, K. S., and Neale, M. C. (2003). Schizophrenia as a complex trait: evidence from a metaanalysis of twin studies. Arch. Gen. Psychiatry 60, 1187-1192.

Tzourio-Mazoyer, N., Landeau, B., Papathanassiou, D., Crivello, F., Etard, O., Delcroix, N., Mazoyer, B., and Joliot, M. (2002). Automated anatomical labeling of activations in SPM using macroscopic anatomical parcellation of the MN MRI single-subject brain. Neuroimage 15, 273-289.

Van den Heuvel, M. P., and Hulshoff Pol, H. E. (2010). Exploring the brain network: a review on resting state fMRI functional connectivity. Eur. Neuropsychopharmacol. 20, 519-534.
Van den Heuvel, M. P., Mandl, R. C., and Hulshoff Pol, H. E. (2008). Normalized group clustering of resting-state fMRI data. PLoS ONE 3, e2001. doi:10.1371/journal.pone.0002001

Van den Heuvel, M. P., Mandl, R. C., Kahn, R. S., and Hulshoff Pol, H. E. (2009). Functionally linked restingstate networks reflect the underlying structural connectivity architecture of the human brain. Hum. Brain Mapp. 30, 3127-3141.

Van den Heuvel, M. P., Mandl, R. C., Stam, C. J., Kahn, R. S., and Hulshoff Pol, H. E. (2010). Aberrant frontal and temporal complex network structure in schizophrenia: a graph theoretical analysis. J. Neurosci. 30, 15915-15926.

Van Gelderen, P., Ramsey, N. F., Liu, G., Duyn, J. H., Frank, J. A., Weinberger, D. R., and Moonen, C. T. W. (1995). Three dimensional functional MRI of human brain on a clinical $1.5 \mathrm{~T}$ scanner. Proc. Natl. Acad. Sci. U.S.A. 92, 6906-6910.

Vollema, M. G., and Ormel, J. (2000). The reliability of the structured interview for schizotypyrevised. Schizophr. Bull. 26 619-629.

Wang, J., Wang, L., Zang, Y., Yang, H., Tang, H., Gong, Q., Chen, Z., Zhu, C., and He, Y. (2009). Parcellation-dependent smallworld brain functional networks: a resting-state fMRI study. Hum. Brain Mapp. 30 1511-1523.

Wernicke, C. (1906). Grundrisse der Psychiatrie. Leipzig: Thieme.

Wig, G. S., Schlaggar, B. L., and Petersen, S. E. (2011). Concepts and principles in the analysis of brain networks. Ann. N. Y. Acad. Sci. 1224 126-146.

Zalesky, A., Fornito, A., and Bullmore, E. T. (2010). Network-based statistic: identifying differences in brain networks. Neuroimage 53, 1197-1207.

Zalesky, A., Fornito, A., Seal, M. L., Cocchi, L., Westin, C. L., Bullmore, E. T. Egan, G. F., and Pantelis, C. (2011). Disrupted axonal fiber connectivity in schizophrenia. Biol. Psychiatry 69, 80-89.
Conflict of Interest Statement: The authors declare that the research was conducted in the absence of any commercial or financial relationships that could be construed as a potential conflict of interest.

Received: 14 September 2011; accepted: 28 November 2011; published online: 16 December 2011.

Citation: Collin G, Hulshoff Pol HE, Haijma SV, Cahn W, Kahn RS and van den Heuvel MP (2011) Impaired cerebellar functional connectivity in schizophrenia patients and their healthy siblings. Front. Psychiatry 2:73. doi: 10.3389/fpsyt.2011.00073

This article was submitted to Frontiers in Neuropsychiatric Imaging and Stimulation, a specialty of Frontiers in Psychiatry. Copyright (c) 2011 Collin, Hulshoff Pol, Haijma, Cahn, Kahn and van den Heuvel. This is an open-access article distributed under the terms of the Creative Commons Attribution Non Commercial License, which permits non-commercial use, distribution, and reproduction in other forums, provided the original authors and source are credited. 